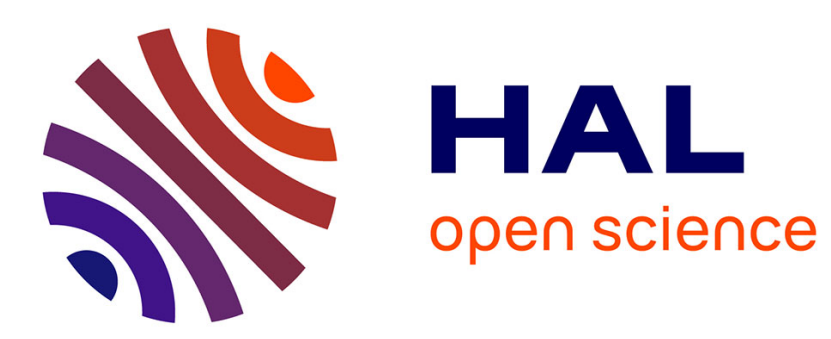

\title{
Contribution vibrationnelle aux propriétés thermodynamiques d'une monocouche physisorbée
}

Bahram Djafari-Rouhani, L. Dobrzynski

\section{To cite this version:}

Bahram Djafari-Rouhani, L. Dobrzynski. Contribution vibrationnelle aux propriétés thermodynamiques d'une monocouche physisorbée. Journal de Physique Lettres, 1976, 37 (9), pp.213-217. 10.1051/jphyslet:01976003709021300 . jpa-00231277

\section{HAL Id: jpa-00231277 https://hal.science/jpa-00231277}

Submitted on 1 Jan 1976

HAL is a multi-disciplinary open access archive for the deposit and dissemination of scientific research documents, whether they are published or not. The documents may come from teaching and research institutions in France or abroad, or from public or private research centers.
L'archive ouverte pluridisciplinaire HAL, est destinée au dépôt et à la diffusion de documents scientifiques de niveau recherche, publiés ou non, émanant des établissements d'enseignement et de recherche français ou étrangers, des laboratoires publics ou privés. 


\title{
CONTRIBUTION VIBRATIONNELLE AUX PROPRIÉTÉS THERMODYNAMIQUES D'UNE MONOCOUCHE PHYSISORBÉE
}

\author{
B. DJAFARI ROUHANI \\ C.E.N. Saclay, Section d'études des interactions gaz-solides \\ B.P. 2, 91190 Gif-sur-Yvette, France
}

et

\section{DOBRZYNSKI}

Laboratoire des Surfaces et Interfaces $\left({ }^{*}\right)$

I.S.E.N., 3 rue F.-Baës, 59046 Lille Cedex, France

(Reçu le 27 avril 1976, accepté le 9 juin 1976)

\begin{abstract}
Résumé. - A l'aide d'un modèle simple, nous calculons analytiquement l'expression en $T^{3}$ de la chaleur spécifique vibrationnelle à basse température $T$ d'une monocouche adsorbée et numériquement les variations de la chaleur spécifique et de l'entropie vibrationnelle à toute température dans le cas d'une couche faiblement liée au substrat. La chaleur spécifique présente un maximum pour une température donnée.
\end{abstract}

\begin{abstract}
Using a simple model for an adsorbed monolayer, we derive analytically the $T^{3}$ vibrational contribution to the low temperature specific heat and compute numerically the vibrational specific heat and entropy of a physisorbed layer for all temperatures. The specific heat exhibits a maximum at a certain temperature.
\end{abstract}

Pour étudier les propriétés vibrationnelles d'une couche adsorbée, il faudrait en principe connaître en premier lieu la structure géométrique d'équilibre (statique) de l'adsorbat par rapport au substrat. Celle-ci dépend des interactions interatomiques ainsi que des distances d'équilibre relatives des atomes du substrat et de ceux de l'adsorbat. Malgré quelques études théoriques $[1,2]$ dans ce domaine, ce problème est loin d'avoir reçu une solution assez générale. Aussi l'on peut étudier les propriétés vibrationnelles soit pour des problèmes spécifiques où l'on connaît expérimentalement la géométrie de l'adsorption [3-5], soit dans des modèles simples où l'on peut dégager l'effet de quelques paramètres caractéristiques du système, tels que les diverses constantes de force ou les masses des atomes, sur ses propriétés vibrationnelles [6]. Dans ce papier nous choisissons un modèle qualitatif et nous nous intéressons plus particulièrement au cas où l'interaction entre le substrat et l'adsorbat peut devenir très faible.

Le substrat est un cristal cubique simple terminé par une surface (001) composé d'atomes de masse $M$. Les interactions interatomiques sont du type de Montroll-Potts [7] avec une constante de force $\beta$

$\left(^{*}\right)$ De la Faculté Libre des Sciences, associé au C.N.R.S. entre atomes premiers voisins. La couche adsorbée est introduite en changeant dans le plan atomique de la surface les paramètres $(M, \beta)$ en $\left(M_{\mathrm{A}}, \beta_{\mathrm{A}}\right)$ et la constante de force entre les deux premiers plans de la surface de $\beta$ en $\beta^{\prime}$. Cette monocouche est donc en épitaxie parfaite par rapport au substrat.

Le modèle de Montroll-Potts a l'inconvénient de ne pas être invariant dans les rotations infinitésimales de l'ensemble du système [8] et de ne pas fournir les ondes de Rayleigh. Cependant il permet en général d'obtenir qualitativement les propriétés vibrationnelles et en particulierle comportement des grandeurs thermodynamiques [6, 9-12].

A l'aide de ce modèle, nous établissons d'abord analytiquement la variation de la chaleur spécifique due à la couche adsorbée à basse température. Nous nous plaçons ensuite dans le cas particulier

$$
\beta^{\prime} \neq \beta=\beta_{\mathrm{A}}, \quad M=M_{\mathrm{A}}
$$

et nous calculons numériquement les variations de la chaleur spécifique $\Delta c_{\mathrm{v}}$ et de l'entropie $\Delta S$ en fonction de la température pour plusieurs valeurs du rapport $\beta^{\prime} \mid \beta$ caractérisant l'interaction de la couche adsorbée avec le substrat. Rappelons que ce même modèle a été utilisé dans la référence [6] pour étudier les propriétés 
thermodynamiques d'une monocouche adsorbée différant du substrat uniquement par la masse des atomes.

Le calcul de ces grandeurs thermodynamiques peut être effectué si l'on connaît la grandeur :

$$
\Delta\left(\omega^{2}\right)=\operatorname{dét}\left(\overleftrightarrow{I}-\overleftrightarrow{V} \overleftrightarrow{G}_{\mathrm{S}}\right)
$$

où $\overleftrightarrow{G}_{\mathrm{S}}$ est la fonction de Green d'un cristal semiinfini terminé par une surface (001) et $V$ la perturbation due à la couche adsorbée. L'expression (1) se calcule aisément par une extension des résultats de la référence [13] qui utilise le modèle précédent pour étudier les déplacements quadratiques moyens des atomes d'une couche physisorbée. Tenant compte de la dégénérescence d'ordre 3 (correspondant aux trois polarisations $x, y, z$ ) des phonons dans le modèle de Montroll-Potts et utilisant une transformation de Fourier parallèle à la surface, l'on peut écrire :

$$
\Delta\left(\omega^{2}\right)=\prod_{i=x, y, z}\left\{\sum_{\varphi_{x}, \varphi_{y}} \Delta_{i}\left(\omega^{2}, \varphi_{x}, \varphi_{y}\right)\right\}
$$

où $\varphi_{i}=k_{i} a$ et $\mathbf{k}=\left(k_{x}, k_{y}\right)$ est un vecteur bidimensionnel dans l'espace réciproque de la surface, et [13]

$$
\begin{aligned}
\Delta_{i}\left(\omega^{2}, \varphi_{x}, \varphi_{y}\right)=1 & +\mu t(2-t)- \\
& -(v-2 \alpha E) \frac{t}{t-1}(1+\mu t) .
\end{aligned}
$$

Nous avons introduit les nouvelles notations :

$$
\begin{aligned}
& \alpha=\frac{M_{\mathrm{A}}-M}{M}, \quad \mu=\frac{\beta^{\prime}-\beta}{\beta}, \\
& v=\frac{2\left(\beta_{\mathrm{A}}-\beta\right)}{\beta}\left(2-\cos \varphi_{x}-\cos \varphi_{y}\right)
\end{aligned}
$$

$t$ est défini par :

$$
t=\left\{\begin{array}{l}
\mathcal{S}-\sqrt{\mathcal{S}^{2}-1} \text { si } \mathcal{S}>1 \\
\mathcal{S}+i \sqrt{1-\mathcal{S}^{2}} \text { si }-1<\mathcal{S}<1 \\
\mathcal{S}+\sqrt{\mathcal{S}^{2}-1} \text { si } \mathcal{S}<-1
\end{array}\right.
$$

où :

$$
\mathcal{S}=3-E-\cos \varphi_{x}-\cos \varphi_{y}
$$

et

$$
E=\frac{M \omega^{2}}{2 \beta}
$$

Nous commençons par le calcul de $\Delta c_{\mathrm{v}}$ à basse température. Celui-ci peut être effectué à partir de la méthode des déphasages [14] (voir ci-dessous). Nous lui préférons une méthode différente, due à Maradudin et al. [15], qui a l'avantage a'éviter la nécessité d'une discussion de l'existence de modes localisés (et éventuellement d'autres discontinuités dans le déphasage) selon les valeurs des paramètres. Cette méthode qui consiste à évaluer la variation d'une grandeur thermodynamique sous la forme d'une intégrale dans le plan complexe, fournit pour l'énergie libre à basse température le développement :

$$
\begin{aligned}
& \Delta F(T)=\Delta E_{0}-\frac{k_{\mathrm{B}} T}{\pi}\left\{\frac{\pi^{2}}{6} \Omega(0) \frac{k_{\mathrm{B}} T}{\hbar}-\right. \\
& \left.-\frac{\pi^{4}}{90} \Omega^{(2)}(0)\left(\frac{k_{\mathrm{B}} T}{\hbar}\right)^{3}+\frac{\pi^{6}}{945} \Omega^{(4)}(0)\left(\frac{k_{\mathrm{B}} T}{\hbar}\right)^{5}+0\left(T^{5}\right)\right\}
\end{aligned}
$$

où

$$
\begin{aligned}
\Omega(y) & =\frac{\mathrm{d}}{\mathrm{d} y}\left\{\left.\log \Delta\left(\omega^{2}\right)\right|_{\omega=i y}\right\} \\
\Omega^{(i)}(0) & =\left.\frac{\mathrm{d}^{i} \Omega(y)}{\mathrm{d} y^{i}}\right|_{y=0}
\end{aligned}
$$

et

$$
\Delta E_{0}=-\frac{\hbar}{2 \pi} \int_{0}^{\infty} y \Omega(y) \mathrm{d} y .
$$

Dans le calcul de $\Delta c_{\mathrm{v}}$ à basse température, seuls les modes de grandes longueurs d'ondes fournissent une contribution. importante. Par ailleurs dans l'expression (6) les $\Omega^{(i)}(y)$ sont considérés dans la limite $y \rightarrow 0$. Aussi, nous développons $\Delta_{i}\left(\omega^{2}, \varphi_{x}, \varphi_{y}\right)$ (éq. 2) dans la limite $\varphi_{x}, \varphi_{y} \ll 1$ et $M \omega^{2} / 2 \beta \ll 1$ :

$$
\Delta_{i}\left(\omega^{2}=-y^{2}, \varphi_{x}, \varphi_{y}\right) \simeq
$$

$$
\simeq(1+\mu)\left[1+\frac{\beta_{\mathrm{A}}-\beta}{\beta} \varphi^{2}+\frac{\alpha M y^{2}}{\beta}\right] \frac{1}{\sqrt{\varphi^{2}+\frac{M y^{2}}{\beta}}}
$$

utilisant (1), (7) et (6) et remarquant que

$$
\Delta c_{\mathrm{v}}(T)=-T \frac{\partial^{2} \Delta F}{\partial T^{2}}
$$

nous obtenons :

$$
\begin{aligned}
\Delta c_{\mathrm{v}}(T)=\frac{2 \pi^{2}}{5} N_{\mathrm{S}} k_{\mathrm{B}} & \left(\frac{k_{\mathrm{B}} T}{\hbar}\right)^{3}\left(\frac{M}{\beta}\right)^{3 / 2} \times \\
& \times\left(3 \alpha+2 \frac{\beta-\beta_{\mathrm{A}}}{\beta}\right)+0\left(T^{3}\right)
\end{aligned}
$$

où $N_{\mathrm{S}}$ est le nombre d'atomes dans un plan (001).

Ce premier terme est d'ordre $T^{3}$ et n'altère donc pas le terme en $T^{2}$ de la chaleur spécifique de surface. Nous pensons que ce résultat est général et apparaîtrait également dans un modèle plus réaliste. Par ailleurs le coefficient du terme en $T^{3}$ dépend de la masse des atomes de la couche adsorbée ainsi que de la constante de force $\beta_{\mathrm{A}}$ à l'intérieur de la couche mais non de la constante de force $\beta^{\prime}$ reliant un atome de l'adsorbat à un atome du substrat. Cette dernière intervient dans le terme suivant d'ordre $T^{5}$, de $\Delta c_{\mathrm{v}}$. Ainsi si l'on se place dans le cas particulier $M=M_{\mathrm{A}}, \beta=\beta_{\mathrm{A}} \neq \beta^{\prime}$ 
et si l'on développe $\Delta_{i}\left(\omega^{2}, \varphi_{x}, \varphi_{y}\right)$ un ordre plus loin qu'en (8) nous obtenons :

$$
\begin{aligned}
& \Delta_{i}\left(\omega^{2}=-y^{2}, \varphi_{x}, \varphi_{y}\right) \simeq \\
\simeq & 1+\mu-\mu\left[\varphi^{2}+\frac{M y^{2}}{\beta}-\left(\varphi^{2}+\frac{M y^{2}}{\beta}\right)^{3 / 2}\right]
\end{aligned}
$$

et

$\Delta c_{\mathrm{v}}(T)=-\frac{8}{7} \pi^{4} N_{\mathrm{S}} \frac{\mu}{1+\mu}\left(\frac{M}{\beta}\right)^{5 / 2} k_{\mathrm{B}}\left(\frac{k_{\mathrm{B}} T}{\hbar}\right)^{5}+0\left(T^{5}\right)$.

D'après (9) et (11), nous observons qu'une diminution des constantes de force ou parallèlement une augmentation de la masse à la surface conduit à une augmentation de la chaleur spécifique et vice versa.

Il est à noter que les développements précédents sont valables dans la mesure où $1+\mu$ n'est pas proche de 0 (c'est-à-dire $\beta^{\prime} \neq 0$ ) : plus $1+\mu$ est proche de 0 , plus la région de température où les lois précédentes sont valables est limitée au voisinage de $0 \mathrm{~K}$.

Enfin ces résultats montrent que pour une couche adsorbée ayant une structure commensurable par rapport au substrat la loi de variation de la chaleur spécifique à basse température est en puissance de $T$ et non en $\mathrm{e}^{-\boldsymbol{A} / \boldsymbol{T} T}$ comme l'ont suggéré quelques auteurs $[2,16]$. Ces derniers calculs sont en effet basés sur un modèle de substrat rigide qui ne satisfait pas à la condition d'invariance par translation de l'ensemble $\mathrm{du}$ système et conduisent en conséquence à une branche de phonons ayant une énergie finie à $\mathbf{k}=0$.

Nous nous intéressons maintenant à l'évolution des grandeurs thermodynamiques avec la température (pour tout $T$ ) lorsque l'interaction entre la couche adsorbée et le substrat peut devenir très faible. C'est pourquoi nous supposons $M_{\mathrm{A}}=M, \beta_{\mathrm{A}}=\beta$ et nous étudions les propriétés de la monocouche lorsque $\beta^{\prime}$ varie et plus spécialement pour $\beta^{\prime} / \beta$ petit. Pour chaque valeur du rapport $\beta^{\prime} / \beta$ nous calculons numériquement $\Delta c_{\mathrm{v}}(T)$ et $\Delta S(T)$ par la méthode des déphasages. Celle-ci consiste à écrire la variation d'une grandeur thermodynamique $F=\sum_{s} f\left(\omega_{s}\right)$ (où la sommation sur $s$ s'étend à tous les modes propres du système) sous la forme [14] :

$$
\Delta F=-\frac{1}{\pi} \int \mathrm{d} \omega \frac{\mathrm{d} f(\omega)}{\mathrm{d} \omega} \eta\left(\omega^{2}\right)
$$

où le déphasage $\eta\left(\omega^{2}\right)$ est défini par :

$$
\eta\left(\omega^{2}\right)=-\operatorname{Arg} \Delta\left(\omega^{2}\right) .
$$

D'après (1) et (2) on peut encore écrire :

$$
\begin{aligned}
\eta\left(\omega^{2}\right)= & 3 \eta_{i}\left(\omega^{2}\right)= \\
& =3 \sum_{\varphi_{x}, \varphi_{y}} \eta_{i}\left(\omega^{2}, \varphi_{x}, \varphi_{y}\right) \quad(i=x, y, z)
\end{aligned}
$$

où

$$
\begin{aligned}
& \eta_{i}\left(\omega^{2}, \varphi_{x}, \varphi_{y}\right)= \\
& \quad=-\operatorname{Arg} \Delta_{i}\left(\omega^{2}, \varphi_{x}, \varphi_{y}\right)=-\operatorname{Arg}\left(1+2 \mu t-\mu t^{2}\right) .
\end{aligned}
$$

Dans l'expression (12), la contribution provenant des états à l'intérieur des bandes de volume et celle provenant des modes localisés doivent être distinguées. Cependant dans notre modèle il ne peut exister que des modes localisés au-dessus des bandes de volume, et ce dans le cas d'un couplage adsorbat-substrat suffisamment fort (en l'occurrence $\beta^{\prime}>\frac{4}{3} \beta$ ). Ce problème ne se pose donc pas pour des valeurs faibles de $\beta^{\prime}$ auxquelles nous nous intéressons.

Etudions d'abord le déphasage qui peut s'écrire d'après (14) et (4) à l'intérieur des bandes de volume :

$$
\eta_{i}\left(\omega^{2}, \varphi_{x}, \varphi_{y}\right)=\operatorname{Arctg}\left\{-\frac{2 \mu \sqrt{1-\delta^{2}}(1-\delta)}{1+\mu+2 \mu \delta(1-\delta)}\right\} \text {. }
$$

Sur la figure $1 a$ nous avons représenté $\eta_{i}\left(\omega^{2}, \varphi_{x}, \varphi_{y}\right)$ pour plusieurs valeurs du rapport $\beta^{\prime} / \beta$ à savoir $\beta^{\prime} \mid \beta=0,5 ; 0,1$ et 0,01 . En abscisses nous avons porté la variable $S$ (éq. (5)) qui prend les valeurs +1 et -1 respectivement en bas et en haut de la bande de volume. La figure $1 b$ représente le déphasage $\eta_{i}\left(\omega^{2}\right)$ (éq. (13)), obtenue à partir de $\eta_{i}\left(\omega^{2}, \varphi_{x}, \varphi_{y}\right)$ par sommation sur
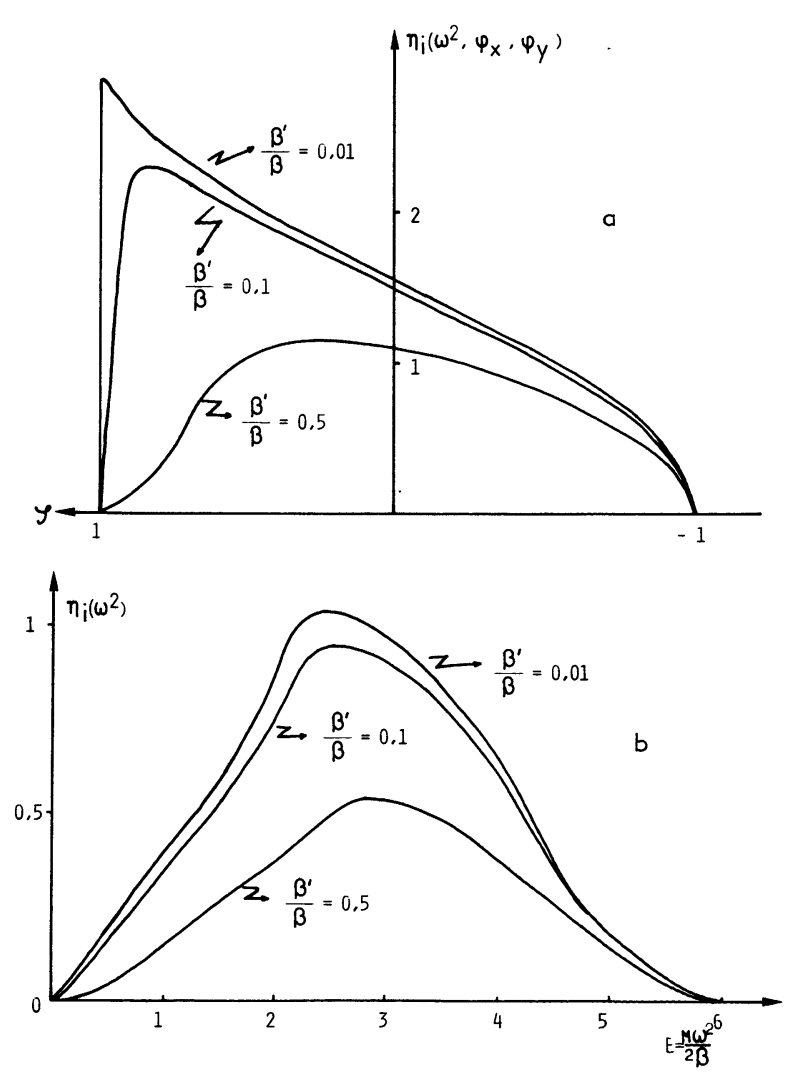

FIG. 1. - Déphasage partiel $\eta_{i}\left(\omega^{2}, \varphi_{x}, \varphi_{y}\right)$ et déphasage total (par atome de surface) $\eta_{i}\left(\omega^{2}\right)$ pour trois valeurs de $\beta^{\prime} / \beta(i=x, y, z$ désigne l'une des trois polarisations). 
$\varphi_{x}$ et $\varphi_{y}$, pour les trois valeurs de $\beta^{\prime} / \beta$ (le résultat est rapporté à un atome de surface). Nous constatons, qu'après sommation sur $\varphi_{x}$ et $\varphi_{y}$, l'allure générale de la courbe de déphasage est identique dans les trois cas ; il y a uniquement variation de l'ordre de grandeur du maximum et un léger déplacement de ce maximum vers les basses énergies lorsque $\beta^{\prime}$ diminue.

Nous pouvons également calculer la variation de la densité d'états

$$
\Delta n\left(\omega^{2}\right)=\frac{1}{\pi} \frac{\mathrm{d} \eta\left(\omega^{2}\right)}{\mathrm{d} \omega^{2}}
$$

par sommation sur $\varphi_{x}$ et $\varphi_{y}$ de la densité d'états partielle :

$$
\begin{aligned}
& \Delta n_{i}\left(\omega^{2}, \varphi_{x}, \varphi_{y}\right)=\frac{1}{\pi} \frac{\mathrm{d} \eta_{i}\left(\omega^{2}, \varphi_{x}, \varphi_{y}\right)}{\mathrm{d} \omega^{2}}=- \\
& -\frac{1}{\pi} \frac{M}{\beta} \frac{\mu(2 \delta+1+3 \mu) \sqrt{\frac{1-\mathcal{S}}{1+\mathcal{S}}}}{4 \mu^{2}\left(1-\mathcal{S}^{2}\right)(1-\mathcal{S})^{2}+[1+\mu+2 \mu \delta(1-\delta)]^{2}}
\end{aligned}
$$

Remarquons simplement que $\Delta n\left(\omega^{2}\right)$ présente des pics en fonction de $\omega^{2}$ mais aucune singularité. On peut se rendre compte facilement de ce dernier point en remarquant que seul le facteur $1 / \sqrt{1+\delta}$ dans (16) peut conduire à une divergence par sommation sur $\varphi_{x}, \varphi_{y}$. Or on peut montrer que cette fonction ne présente pas une telle divergence lors de l'intégration sur $\varphi_{x}$ et $\varphi_{y}$.

Utilisant les expressions (12)-(15) nous avons calculé, pour les trois valeurs précédentes de $\beta^{\prime} / \beta$, les variations de $\Delta c_{\mathrm{v}}(T)$ et $\Delta S(T)$ en fonction de la température. Les résultats rapportés à un atome de surface sont représentés sur les figures $2 . \Delta c_{\mathrm{v}}$ présente un maximum. Ce comportement s'explique par le fait que pour $T=0$ et pour $T / T_{\mathrm{D}} \gg 1$ (où $T_{\mathrm{D}}$ est défini par :

$$
k T_{\mathrm{D}}=\hbar \omega_{\mathrm{M}}=\hbar \sqrt{\frac{12 \beta}{M}}
$$

où $\omega_{\mathrm{M}}$ est la fréquence maximum de la bande de volume) cette quantité doit s'annuler. L'entropie $\Delta S$ qui se déduit de $\Delta c_{\mathrm{v}}$ par intégration

$$
\left(\Delta S(T)=\int_{0}^{T} \frac{\Delta c_{\mathrm{v}}\left(T^{\prime}\right)}{T^{\prime}} \mathrm{d} T^{\prime}\right)
$$

tend donc vers un palier lorsqu'on augmente la température. Nous pouvons conclure d'après cette étude que le passage d'une valeur de $\beta^{\prime} / \beta$ à une autre modifie l'ordre de grandeur des résultats mais ne change pas l'allure des courbes (même en ce qui concerne la position des maxima sur la figure $2 a$ ).
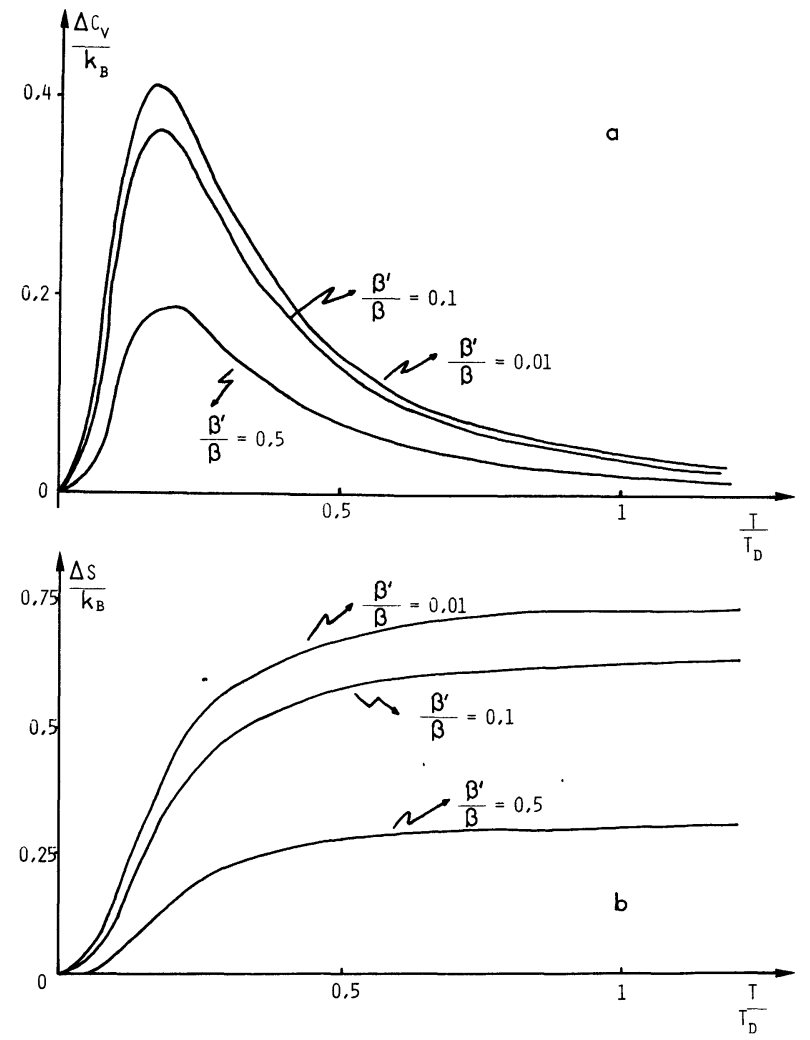

FIG. 2. - Variations de la chaleur spécifique et de l'entropie par atome adsorbé en fonction de la température pour trois valeurs de $\beta^{\prime} / \beta$.

Ce comportement résulte du fait que l'allure du déphasage $\eta_{i}\left(\omega^{2}\right)$ (Fig. 1b) dépend elle-même peu de $\beta^{\prime} / \beta$. L'allure des courbes de $\Delta c_{\mathrm{v}}(T)$ et de $\Delta S(T)$ obtenues ici est qualitativement semblable à celle des courbes obtenues pour une variation de masse [6] $\left(M_{\mathrm{A}} \neq M\right)$ et serait aussi comparable pour une variation $\beta_{\mathrm{A}} \neq \beta$.

Enfin rappelons que dans les calculs précédents, nous avons introduit la couche adsorbée en changeant les paramètres au voisinage de la surface. Si on l'introduit plutôt en ajoutant une monocouche d'atomes de l'adsorbat au substrat semi-infini, les variations des grandeurs thermodynamiques s'obtiennent en ajoutant aux résultats précédents la contribution d'une monocouche d'atomes en volume.

Note ajoutée aux épreuves. - Si l'on désigne par $C_{\mathrm{v}}^{0}$ la chaleur spécifique à basse température de $N_{\mathrm{s}}$ atomes en volume [17], l'expression (9) peut s'écrire :

$$
\Delta C_{\mathrm{v}}=C_{\mathrm{v}}^{0}\left(3 \alpha+2 \frac{\beta-\beta_{\mathrm{A}}}{\beta}\right) \text {. }
$$

Lorsque $\Delta C_{\mathrm{v}}$ est négatif, la variation totale de la chaleur spécifique à basse température lors de l'adsorption, à savoir $C_{\mathrm{v}}^{0}+\Delta C_{\mathrm{v}}$, peut dans certains cas devenir négative. Un résultat semblable a été aussi trouvé dans un calcul numérique récent [18] pour certains systèmes gaz rares/gaz rares. 


\section{Bibliographie}

[1] Frank, F. C., van der Merwe, J. H., Proc. R. Soc. A 198 (1949) 205 et 216, ibid. A 200 (1949) 125.

[2] Ying, S. C., Phys. Rev. B 3 (1971) 4160.

[3] Allen, R. E., Alldredge, G. P., De Wette, F. W., J. Chem. Phys. 54 (1971) 2605.

[4] Theeten, J. B., Armand, G., Phys. Rev. 9 (1974) 3969.

[5] Suzanne, J., Masri, P., Bienfait, M., Surf. Sci. 43 (1974) 441.

[6] Dobrzynski, L., Mills, D. L., J. Phys. \& Chem. Solids 30 (1969) 1043.

[7] Montroll, E. W., Potts, R. B., Phys. Rev. 100 (1955) 525.

[8] Ludwig, W., Lengeler, B., Solid State Commun. 2 (1964) 83.

[9] Dobrzynski, L., Leman, G., J. Phys. A 30 (1969) 119.

[10] Cunningham, S. L., Surf. Sci. 33 (1972) 139.
[11] Cunningham, S. L., Dobrzynski, L., Maradudin, A. A., Phys. Rev. B 7 (1973) 4643.

[12] Dobrzynski, L., Mills, D. L., Phys. Rev. B 7 (1973) 1322.

[13] Dobrzynski, L., Lajzerowicz, J., Phys. Rev. B 12 (1975) 1358.

[14] Lenglart, P., Dobrzynski, L., Leman, G., Annls de Phys. 7 (1972) 407

[15] Maradudin, A. A., Montroll, E. W., Weiss, G. H., IPaTova, I. P., Solid State Phys. Suppl. 3 (1971) 390.

[16] Das, J. G., Prog. Surf. Membr. Sci. 7 (1973) 95.

[17] Landau, L. D., Lifshitz, E. M., Statistical Physics (Pergamon Press), 1959, p. 184.

[18] LaWrence, W. H., Allen, R. E., Phys. Rev. (à paraître). 